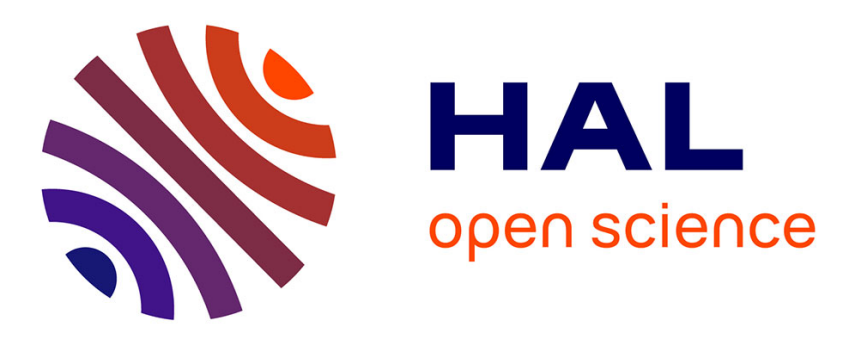

\title{
Low ML-detection complexity, adaptive 2x2 STBC, with powerful FEC codes
}

\author{
Ammar El Falou, Charlotte Langlais, Charbel Abdel Nour, Catherine
}

Douillard

\section{- To cite this version: \\ Ammar El Falou, Charlotte Langlais, Charbel Abdel Nour, Catherine Douillard. Low ML- detection complexity, adaptive 2x2 STBC, with powerful FEC codes. 7th International Sym- posium on Turbo Codes \& Iterative Information Processing, Aug 2012, Gothenburg, Sweden. 10.1109/ISTC.2012.6325226 . hal-00797564}

\section{HAL Id: hal-00797564 \\ https://hal.science/hal-00797564}

Submitted on 23 Jun 2021

HAL is a multi-disciplinary open access archive for the deposit and dissemination of scientific research documents, whether they are published or not. The documents may come from teaching and research institutions in France or abroad, or from public or private research centers.
L'archive ouverte pluridisciplinaire HAL, est destinée au dépôt et à la diffusion de documents scientifiques de niveau recherche, publiés ou non, émanant des établissements d'enseignement et de recherche français ou étrangers, des laboratoires publics ou privés. 


\title{
Low ML-detection complexity, adaptive $2 \times 2$ STBC, with powerful FEC codes
}

\author{
A. El Falou, C. Langlais, C. Abdel Nour and C. Douillard \\ Institut Telecom; Telecom Bretagne; UMR CNRS 3192 Lab-STICC \\ Electronics Department, Technopôle Brest Iroise CS 83818, 29238 Brest Cedex 3 \\ Université européenne de Bretagne, France \\ Email: \{ammar.elfalou, charlotte.langlais, charbel.abdelnour, catherine.douillard\}@ telecom-bretagne.eu
}

\begin{abstract}
We present an adaptive space time block code (STBC) for $2 \times 2$ MIMO systems designed to be used with powerful forward error correcting (FEC) codes and having a low ML-detection complexity. STBCs are commonly designed according to the rank-determinant criteria, suitable for high signal to noise ratio (SNR) values. However, powerful FEC codes like turbo codes achieve iterative convergence at low to moderate SNR. Thus, we first propose a non-asymptotic STBC design criterion based on the bitwise mutual information (BMI) maximization at a specific target SNR. According to this criterion, an adaptive matrix D based STBC is defined for a wide range of SNRs. Resulting code outperforms the original matrix $D$ and closes the gap to the more complex Golden code in the context of a WiMAX system.
\end{abstract}

\section{INTRODUCTION}

Multiple input multiple output (MIMO) systems which consist of using multiple antennas at both transmitter and receiver have been adopted in most of the recently developed wireless communication systems, e.g., WiMAX, Wi-Fi, LTE, etc. Indeed, Alamouti code [1] has been incorporated for diversity gain, spatial multiplexing [2] for multiplexing gain and space time block codes (STBCs) make various tradeoffs between them [3].

Full-rate full-diversity STBCs for MIMO systems are conventionally designed according to the well-known asymptotic rank-determinant criteria [4]. Based on the minimization of the pairwise error probability (PEP) between transmitted and detected codewords, these criteria aim to maximize the diversity gain and the coding gain of designed STBCs at high signal to noise ratio (SNR). For $2 \times 2$ MIMO systems, several fullrate full-diversity STBCs have been proposed (see [5-7] and references therein) trading off complexity and bit error rate (BER) performance. At high SNR, the Golden code (GC) [6] is favored thanks to its best coding gain. However, it suffers from a high maximum-likelihood (ML) detection complexity. Motivated by the reduction of this ML-detection complexity, Sezginer and Sari [5] have proposed a full-rate full-diversity STBC called matrix D (MD). Besides, its lower coding gain leads to a slight loss in performance compared to the GC [7].

STBCs designed to perform well at high SNRs do not guarantee a good behavior at low SNRs. Powerful forward error correction (FEC) codes, usually present in the transmission chain, achieve iterative convergence at low to moderate SNRs. FEC decoding is extremely sensitive to its input bitwise mutual information (BMI) value. The higher the BMI at the input of the FEC decoder, the lower the error rate at the output of the decoder. Our work focuses on the design of a STBC for a practical communication system trading off good performance and low detection complexity.

In this paper, we first present a non-asymptotic MIMO STBC design criterion based on the BMI maximization between transmitted and soft detected bits for a specific target SNR. Based on the proposed BMI criterion, we compute the design parameter i.e., the appropriate angle, maximizing the BMI at the matrix D output for each SNR. This leads to the proposal of a low ML-detection complexity, adaptive $M D$ optimized for a wide range of SNRs typical of the use of an adaptive modulation and coding (AMC) scheme. BER simulation results are given for a WiMAX transmission with different AMC schemes and $2 \times 2$ MIMO profiles.

The remainder of this paper is organized as follows. In Section II, the practical communication MIMO system model under consideration is presented. In Section III, the conventional STBC design criteria and the proposed non-asymptotic BMI criterion are given. The adaptive MD STBC is presented in Section IV. Receiver complexity and BER curves are provided in Section V. Section VI concludes the paper.

\section{SySTEM MODEL AND NOTATIONS}

We consider a MIMO system with $N_{t}$ transmit antennas, $N_{r}$ receive antennas operating over a quasi-static flat Rayleigh fading channel, i.e., the channel changes for each transmitted MIMO codeword of length $T$. A perfect channel state information (CSI) is assumed at the receiver, but not at the transmitter. The system is described as follows (see Fig. 1):

Transmitter side: the information word $\mathrm{b}$ is encoded via $\mathrm{a}$ powerful FEC code $\mathrm{C}$ with rate $R_{c}$. The resulting codeword is interleaved with a random interleaver $\Pi$ following a bit interleaved coded modulation (BICM) scheme [8]. We denote by $\mathbf{c}$ the interleaved codeword. c is mapped onto a Gray encoded $M$-QAM constellation where $M=2^{m}$ is the modulation order and $m$ denotes the number of bits per symbol. A MIMO system is said to be full-rate (FR) when the number of transmitted symbols per channel use is equal to the number of transmit antennas $N_{t}$. The mapper feeds a block of $N_{t} \times T$ QAM symbols denoted by $S_{i}$ to the STBC MIMO encoder. 


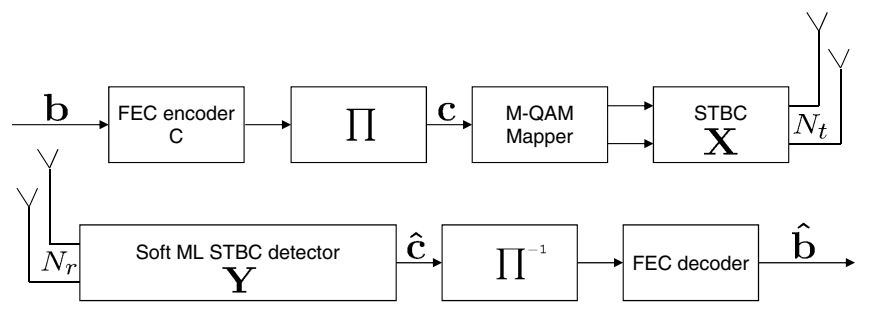

Fig. 1. Structure of the system at the transmitter and the receiver.

Afterwards, encoder output denoted by $\mathbf{X}_{\left[N_{t} \times T\right]}$ is transmitted via $N_{t}$ antennas over $T$ channel use periods.

The MIMO channel is completely defined by the matrix $\mathbf{H}_{\left[N_{r} \times N_{t}\right]}$ where its entries are assumed to be independent and identically distributed (i.i.d.) circularly symmetric Gaussian random variables with probability density function (pdf) $\sim C N(0,1) . C N\left(\mu, \sigma^{2}\right)$ denotes the complex Gaussian pdf with mean $\mu$ and variance $\sigma^{2}$. A complex white Gaussian noise $\mathbf{N}_{\left[N_{r} \times T\right]}$ with i.i.d. entries and pdf $\sim C N\left(0, \sigma^{2}\right)$ is added to the received signals.

The channel input-output relation is given by:

$$
\mathbf{Y}=\mathbf{H X}+\mathbf{N}
$$

Receiver side: $N_{r} \times T$ noisy signals, denoted by $\mathbf{Y}_{\left[N_{r} \times T\right]}$, are received on the $N_{r}$ antennas. A soft ML STBC detector estimates the transmitted $M$-QAM symbols and feeds the $P\left(c_{i}=1\right) \quad\left(c_{i}\right.$ is the $i$-th bit of codeword $\left.\mathbf{c}\right)$, denoted by $\hat{c}_{i}$, to the deinterleaver. These probabilities are deinterleaved then provided to the FEC decoder. Resulting hard decoded information word $\hat{b}$ is compared to $\mathbf{b}$ to compute the BER.

\section{BITWISE MUTUAL INFORMATION CRITERION}

In this section, we first give a brief review of conventional STBC design criteria. Then we present the bitwise mutual information criterion.

\section{A. Conventional STBC design criteria}

The minimization of the union bound for the PEP leads to the well-known rank-determinant STBC design criteria [4]:

1) Rank Criterion: To achieve the maximum diversity, the rank $r$ of the codeword difference matrix $\Delta=\mathbf{X}-\hat{\mathbf{X}}$ must be maximized for all possible transmitted codeword pairs $(\mathbf{X}, \hat{\mathbf{X}})$. The diversity gain is defined as $d=r N_{r}$. When $r=N_{t}$ the system is said to be full-diversity (FD).

2) Determinant Criterion: The STBC minimum determinant is defined as:

$$
\delta=\min _{\mathbf{X} \neq \hat{\mathbf{X}}} \prod_{i=1}^{r} \lambda_{i}
$$

where $\lambda_{i} ; i=1, \ldots, r$ are the non-zero eigenvalues of the matrix $\Delta \Delta^{H}\left(\Delta^{H}\right.$ is the Hermitian of $\left.\Delta\right)$.

In order to obtain the best performance at high SNR values, $\delta$ should be maximized. Indeed, the dominant parameter is the diversity gain $d$ which defines the slope of BER curves. Therefore, it is important to ensure the FD of the STBC and then maximize its coding gain $\delta^{1 / N_{t}}$

\begin{tabular}{|c|c|}
\hline $\begin{array}{c}\text { MIMO Code } \\
\mathbf{X}\end{array}$ & $\begin{array}{c}\text { Minimum determinant } \\
\text { for QAM modulations }\end{array}$ \\
\hline \hline Golden code (GC) & 3.2000 \\
\hline Srinath-Rajan (SR) & 3.2000 \\
\hline Dayal-Varanasi (DV) & 3.2000 \\
\hline Trace-orthonormal (TO) & 3.2000 \\
\hline HTW-PGA & 2.2857 \\
\hline Sezginer-Sari (MD) & 2.0000 \\
\hline Yao-Wornell (YW) & 0.8000 \\
\hline Spatial multiplexing (SM) & 0.0000 \\
\hline
\end{tabular}

TABLE I

COMPARISON BETWEEN THE MINIMUM DETERMINANT OF SOME WELL-KNOWN FULL-RATE $2 \times 2$ STBCS.

\section{B. BMI criterion}

The asymptotic rank-determinant criteria optimize STBCs for high SNRs. Most of practical communication systems are planned for low to moderate target SNRs due to the introduction of a powerful FEC code. Therefore, the STBC parameter optimization should target this SNR range. Based on the maximization of the BMI between transmitted and soft detected bits, we propose a non-asymptotic design criterion aiming at choosing the appropriate design parameter value for STBCs at a specific target SNR.

1) Definition: At a SNR value, the BMI is computed as in [9], by:

$$
\begin{array}{r}
\operatorname{BMI}(\hat{c} ; c)=1-E\left[\log _{2}(1+\exp (-L))\right] \\
\approx 1-\frac{1}{N} \sum_{n=1}^{N} \log _{2}\left(1+\exp \left(-u_{n} . L_{n}\right)\right) \\
\text { with }\left\{\begin{array}{lll}
L_{n}= & \ln \frac{1-\hat{c}_{n}}{\hat{c}_{n}} \\
u_{n}= & (-1)^{c_{n}}
\end{array}\right.
\end{array}
$$

where $E$ is the mean function and $L$ denotes the log-likelihood ratio (LLR). $N$ is assumed to be large enough to accurately estimate the BMI. The BMI value is assessed by Monte Carlo simulations by passing a $N$-bit sequence into the mapper, the STBC, the MIMO channel and the soft ML detector.

The application of the BMI criterion to any $N_{t} \times N_{r}$ STBC is straightforward, we restrict our study to a $2 \times 2$ MIMO system.

2) Validation: For the $2 \times 2$ MIMO system, several FR-FD STBCs have been proposed ([5-7] and references therein). Table I summarizes their characteristics. The minimum determinant is computed for symbols chosen from a regular $M$ QAM constellation where the difference between real part or imaginary part of any two constellation points is a multiple of 2. The minimum determinant provides the MIMO coding gain. At high SNR, the GC [6] (and hence SR, DV and TO STBCs) is known to be the best FR-FD STBC as it has the highest coding gain.

In order to validate the BMI criterion from a MIMO coding gain point of view, we have plotted in Fig. 2 the BMI of STBCs presented in Table I, for a 4-QAM modulation, as a function of high $E_{b u} / N_{0}$ values, where $E_{b u}$ denotes the energy per bit for uncoded MIMO system and $N_{0}$ the noise power spectral 


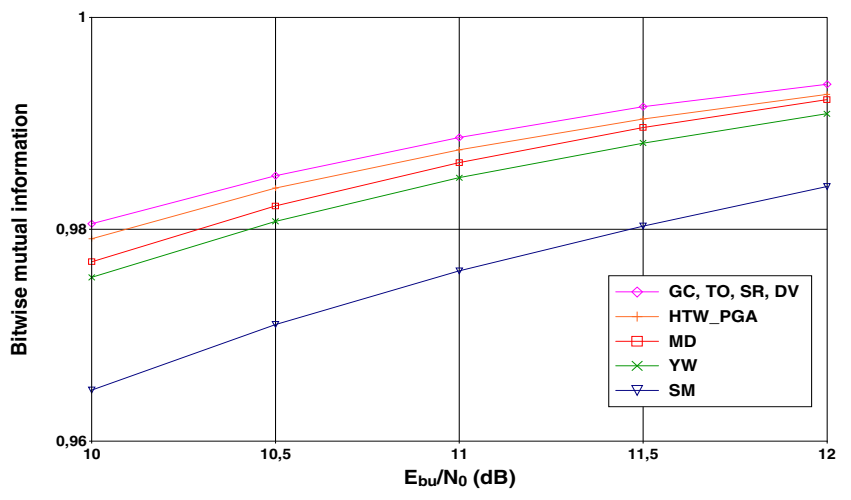

Fig. 2. BMI of some well-known $2 \times 2$ MIMO STBCs for high $E_{b u} / N_{0}$; 4-QAM modulation.

density. We use the expression "uncoded" to refer to the case of a MIMO STBC system without any FEC code. SR, DV and TO STBCs show a quasi identical performance than the GC performance. Fig. 2 shows that the hierarchy of the presented MIMO codes in terms of BMI is identical to their classification in Table I which validates the BMI criterion.

\section{WiMAX $2 \times 2$ MIMO profiles}

As an example of a $2 \times 2$ MIMO system, we consider the worldwide interoperability for microwave access (WiMAX) system which uses the MIMO codes specified in the IEEE 802.16e-2005 standard [10]. Two mandatory MIMO profiles are described for the downlink. The first represents the Alamouti code introduced for transmit diversity, referred to as matrix A (MA). It offers FD but is only half-rate. The second profile is the FR spatial multiplexing introduced for spectral efficiency increase, referred to as matrix B (MB). In order to benefit from both diversity and multiplexing gains, another MIMO profile is included in the IEEE 802.16e-2005 specification, referred to as matrix $\mathrm{C}$ (MC) which is a variant of the GC.

Motivated by the proposal of a FR-FD STBC with reduced complexity, authors in [5] have proposed the matrix D STBC where its design parameter has been optimized according to the rank-determinant criteria. Original detector provides hard decisions on received bits but not soft ones. In [11], we have proposed a soft detector for the MD STBC with the same complexity order.

\section{AdAPTIVE MATRIX D STBC}

In the sequel, the MD optimization according to the BMI criterion is given. Resulting BMI-optimized MD is compared to the original MD and to the other WiMAX MIMO profiles, i.e, the MA, the MB and the MC.

A group of 4 data symbols $\left(S_{1}, S_{2}, S_{3}, S_{4}\right)$ belonging to the complex modulation constellation is transmitted by $N_{t}=2$ antennas, during $T=2$ channel use periods, as follows [5]:

$$
\mathbf{X}^{\mathrm{MD}}=\left[\begin{array}{cc}
a S_{1}+b S_{3} & -c S_{2}^{*}-d S_{4}^{*} \\
a S_{2}+b S_{4} & c S_{1}^{*}+d S_{3}^{*}
\end{array}\right]
$$

Where $a=c=1 / \sqrt{2} ; b=1 / \sqrt{2} \exp (i \varphi) ; d=b \exp \left(-\frac{i \pi}{2}\right)$. $S^{*}$ designates the complex conjugate of $S$ and $\varphi=\arg (b)$ is

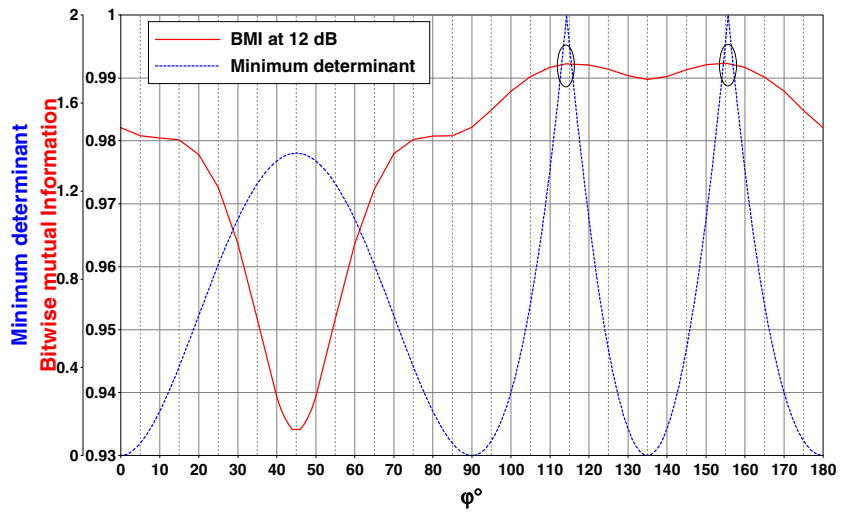

Fig. 3. BMI at $E_{b u} / N_{0}=12 \mathrm{~dB}$ and minimum determinant of MD STBC as a function of its design parameter $\varphi$; 4-QAM modulation.

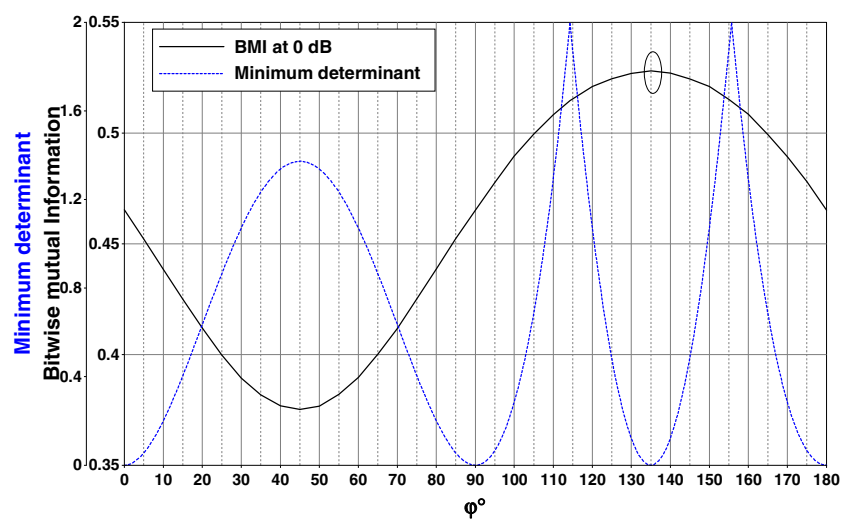

Fig. 4. BMI at $E_{b u} / N_{0}=0 \mathrm{~dB}$ and minimum determinant of MD STBC as a function of its design parameter $\varphi$; 4-QAM modulation.

the code design parameter to be optimized according to the selected criterion.

In [5], an exhaustive search is performed in order to maximize the minimum determinant. This search leads to $\varphi=\arg \left([(1-\sqrt{7}+i(1+\sqrt{7})] /(4 \sqrt{2})) \approx 114.29^{\circ}\right.$. Original MD guarantees a good performance at high SNRs, but not necessarily at low and moderate SNRs regarding the used optimization criteria.

In Fig. 3 and Fig. 4, the MD STBC minimum determinant and the BMI are plotted as a function of the design parameter $\varphi$, for a 4-QAM modulation, for $E_{b u} / N_{0}=12 \mathrm{~dB}$ and $E_{b u} / N_{0}=0 \mathrm{~dB}$ respectively. At high $E_{b u} / N_{0}$ (see Fig. 3), the obtained $\varphi$ under the BMI criterion is equal to $114.29^{\circ}$ as the original one in [5]. While at low $E_{b u} / N_{0}$, Fig. 4 shows that the angle which maximizes the BMI is equal to $\varphi=135^{\circ}$, different from the original one obtained under the determinant criterion. These results show that the MD design parameter value is not unique for both low and high SNRs. The $\varphi$ value is a function of $E_{b u} / N_{0}$. As presented in Fig. 3 and Fig. 4 for $E_{b u} / N_{0}=12 \mathrm{~dB}$ and $E_{b u} / N_{0}=0 \mathrm{~dB}$ respectively, we have computed for each $E_{b u} / N_{0}$ with a step of $0.25 \mathrm{~dB}$, the appropriate $\varphi$ which maximizes its BMI denoted by $\varphi_{\mathrm{opt}}^{M \text {-QAM }}$

For 4-QAM modulation, for the range $5.5 \mathrm{~dB}<E_{b u} / N_{0}<$ $11 \mathrm{~dB}$, and thanks to a polynomial interpolation, the analytical approximation of $\varphi$ versus $E_{b u} / N_{0}$, according to the BMI 
criterion, is given by:

$\varphi_{\mathrm{opt}}^{4-\mathrm{QAM}}=\left\{\begin{array}{c}135^{\circ} ; \text { For } E_{b u} / N_{0} \leq 5.5 \mathrm{~dB} \\ -0.46\left(\frac{E_{b u}}{N_{0}}\right)^{3}+11.92\left(\frac{E_{b u}}{N_{0}}\right)^{2}-102.64\left(\frac{E_{b u}}{N_{0}}\right) \\ +414 ; \text { For } 5.5<E_{b u} / N_{0}<11 \mathrm{~dB} \\ 114.29^{\circ} ; \text { For } E_{b u} / N_{0} \geq 11 \mathrm{~dB}\end{array}\right.$

Similarly, for 16-QAM modulation, the design parameter $\varphi_{\mathrm{opt}}^{16-\mathrm{QAM}}$ is computed by:

$\varphi_{\mathrm{opt}}^{16-\mathrm{QAM}}=\left\{\begin{array}{c}135^{\circ} ; \text { For } E_{b u} / N_{0} \leq 11 \mathrm{~dB} \\ -0.0973\left(\frac{E_{b u}}{N_{0}}\right)^{3}+3.92\left(\frac{E_{b u}}{N_{0}}\right)^{2}-54.05\left(\frac{E_{b u}}{N_{0}}\right) \\ +379.58 ; \text { For } 10<E_{b u} / N_{0}<17 \mathrm{~dB} \\ 114.29^{\circ} ; \text { For } E_{b u} / N_{0} \geq 17 \mathrm{~dB}\end{array}\right.$

In [11], the angle $\varphi=135^{\circ}$ has already been proposed for the MD STBC according to the trace criterion known as a STBC design criterion for low SNRs [12].

For higher order modulations, the same method can be applied and a suitable $\varphi_{\mathrm{opt}}^{M-\mathrm{QAM}}$ can be chosen. The BMI criterion always provides the best choice for the STBC design parameter since it maximizes the BMI at each SNR. An analytical method to get $\varphi_{\mathrm{opt}}^{M-\mathrm{QAM}}$ is still an open problem.

\section{RECEIVER COMPLEXITY AND SIMULATION RESULTS}

\section{A. Adaptive MD parameter selection}

For the WiMAX system, the used FEC code is a 8-state double binary turbo code, with different rates $R_{c}=1 / 2$, $2 / 3,3 / 4$ and $5 / 6$ and a maximal information frame size $K=4,800$ bits. For the adaptive MD STBC, the appropriate $\varphi_{\text {opt }}^{M-Q A M}$ depends on the coding rate $R_{c}$ and the modulation order $M$. This $\varphi_{\mathrm{opt}}^{M-\mathrm{QAM}}$ value is computed as follows:

1) Passing from global system to uncoded MIMO by: $E_{b u} / N_{0}=E_{b} / N_{0}+10 \log _{10}\left(R_{c}\right)$ where $E_{b}$ is the energy per information bit.

2) The obtained $E_{b u} / N_{0}$ is introduced in equation (5) and (6) to compute the value of $\varphi$ to be used for the corresponding coding rate, modulation order and $E_{b} / N_{0}$.

\section{$B$. On the receiver complexity}

A comparison of the receiver complexity between the WiMAX MIMO profiles is assessed by evaluating the MLdetector and the FEC decoder complexities.

1) MIMO detection complexity: The ML-detection complexity of STBCs is conventionally assessed by the order of the number of computed distances required to estimate the transmitted STBC [7]. FEC decoding needs the probability on coded bits $P\left(c_{i}=1\right)$ at its input. Then, a more realistic complexity study should take into account the number of required multiplication and addition per computed $P\left(c_{i}=1\right)$. Table II provides the ML-detection complexity required for soft-decoding one bit (of the coded frame) at the output of MIMO detector, in terms of number of floating point (FP) additions and multiplications for any complex symbols. For square QAM, this complexity can be further reduced, as in [7], for all the presented codes by decoding independently real and imaginary parts of some symbols.

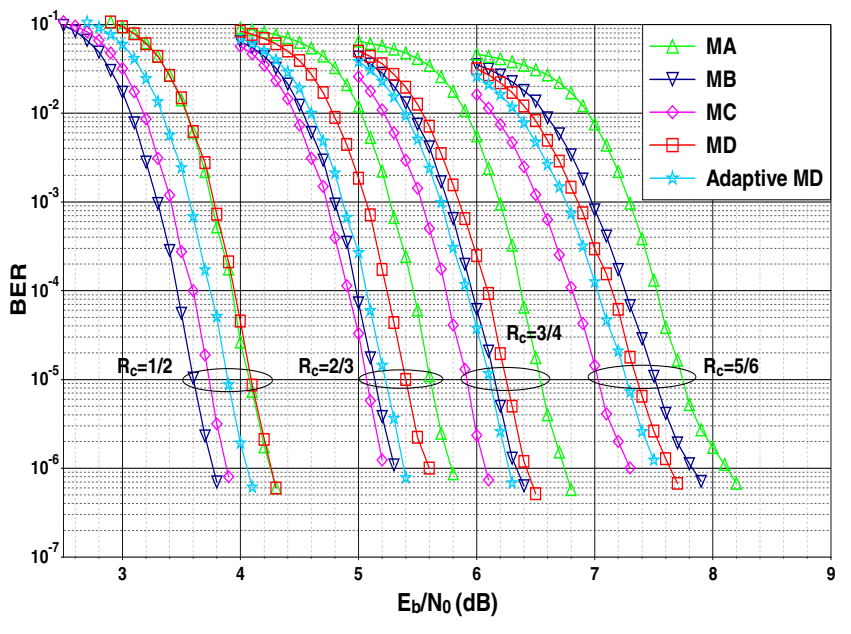

Fig. 5. BER of turbo coded MIMO system; 8 -state double binary TC ( $R_{c}=$ $1 / 2,2 / 3,3 / 4,5 / 6)$ with 8 turbo-decoding iterations; MIMO profiles: MA with 16-QAM and MB, MC, MD, adaptive MD with 4-QAM; quasi-static flat Rayleigh fading MIMO channel.

2) Average number of iterations per frame: As done in [13] for the binary turbo-code, the Max-Log-MAP based turbo decoding requires $N_{\text {symb }}=3.2^{\nu+o}+5.2^{o}+2^{p+2}-9$ floating point additions for the processing of a turbo code information symbol per iteration, where $\nu=3$ denotes the code memory, $o=2$ is the number of information bits per turbo code information symbol and $p=4$ is the number of coded bits provided by the encoder at each trellis stage, leading to $N_{\text {symb }}=171$ for the 8 -state double binary turbo code. Therefore, the FEC decoding complexity per information bit is assessed by $\frac{\bar{N} \cdot N_{\text {symb }}}{o}$ where $\bar{N}$ is the average number of turbodecoding iterations required to correctly decode a received frame. Indeed, $\bar{N}$ is computed by:

$$
\bar{N}=\frac{\sum_{p=1}^{P} n_{p}}{P}
$$

Where $P$ is the number of simulated frames, $n_{p} \leq N_{\max }$ is the number of iterations per frame $p$ required for correct decoding and $N_{\max }$ is the maximum number of turbo code iterations.

The overall complexity per information bit is computed by adding the ML-detection divided by the code rate $R_{c}$ and the FEC decoding complexity.

\section{Simulation results}

BER curves are plotted for an information frame size equal to 4,800 , after 8 turbo-decoding iterations as a function of $E_{b} / N_{0}$ for a transmission over a quasi-static flat Rayleigh fading MIMO channel. A random BICM interleaver is used. The number of transmit antennas is $N_{t}=2$ and receive antennas is $N_{r}=2$. A fair comparison between full-rate MIMO codes and the half-rate MA code is done at the same spectral efficiency. Therefore, we use a $M$-QAM modulation for the FR profiles and $M^{2}$-QAM modulation for the MA. We evaluate the gains at a target $\mathrm{BER}=10^{-5}$.

Fig. 5 shows the BER curves with a 4-QAM modulation for the FR profiles. For $R_{c}=1 / 2$, the MB offers the best performance. From $R_{c}=2 / 3$ to $R_{c}=5 / 6$, the MC surpasses 


\begin{tabular}{|c|c|c|c|c|c|c|}
\hline MIMO profiles & FP addition & FP multiplication & \multicolumn{2}{|c|}{ 4-QAM } & \multicolumn{2}{|c|}{$16-$ QAM } \\
\cline { 3 - 7 } & & & FP add. & FP mul. & FP add. & FP mul. \\
\hline MA / Alamouti & $\frac{16 M+M \log _{2}(M)+11}{\log _{2}(M)}$ & $\frac{24 M+\log _{2}(M)+8}{\log _{2}(M)}$ & 41.5 & 53 & 82.75 & 99 \\
\hline MB / SM & $\frac{38 M^{2}+4 N_{t} M \log _{2}(M)}{2 N_{t} \log _{2}(M)}$ & $\frac{40 M^{2}+2 N_{t} \log _{2}(M)}{2 N_{t} \log _{2}(M)}$ & 84 & 81 & 640 & 641 \\
\hline MD [11] & $\frac{4 M^{3}+190 M^{2}+4 N_{t} M \log _{2}(M)+11}{2 N_{t} \log _{2}(M)}$ & $\frac{M^{3}+250 M^{2}+2 N_{t} \log _{2}(M)+8}{2 N_{t} \log _{2}(M)}$ & 421.375 & 510 & $4,096.68$ & $4,257.5$ \\
\hline MC / GC & $\frac{36 M^{4}+8 N_{t} M \log _{2}(M)+2}{2 N_{t} \log _{2}(M)}$ & $\frac{40 M^{4}+8 N_{t} \log _{2}(M)+3}{2 N_{t} \log _{2}(M)}$ & $1,168.25$ & $1,2844.375$ & 147,520 & 163,844 \\
\hline
\end{tabular}

TABLE II

ML-DETECTION COMPLEXITY PER CODED BIT OF $2 \times 2$ WIMAX MIMO PROFILES IN TERMS OF FLOATING POINT ADDITIONS AND MULTIPLICATIONS.

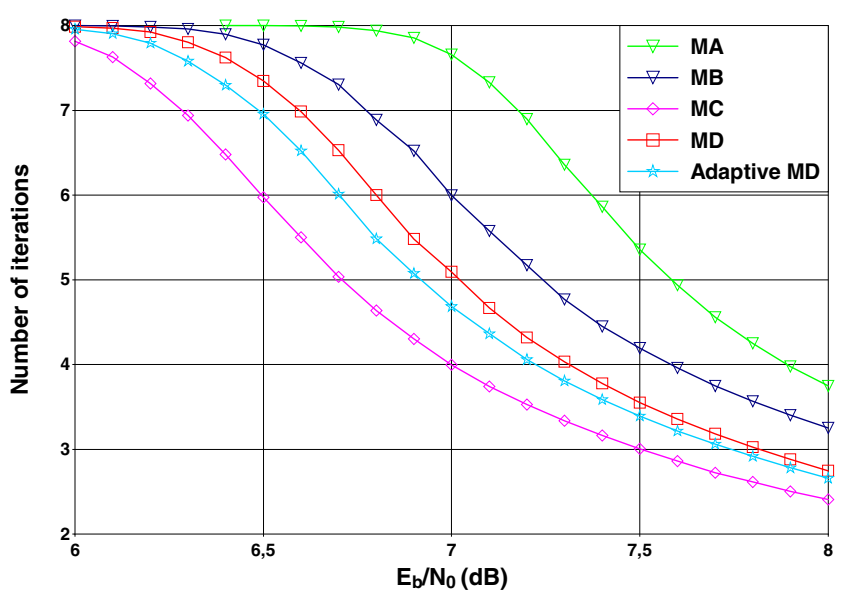

Fig. 6. Average number of turbo-decoding iterations per frame for $R_{c}=5 / 6$; MIMO profiles: MA (16-QAM) and MB, MC, MD, adaptive MD (4-QAM).

all the presented codes. The MA always shows the worst performance. The proposed adaptive MD always overcomes the original MD with a gain between 0.1 and $0.2 \mathrm{~dB}$ while a loss less than $0.2 \mathrm{~dB}$ is observed with respect to the MC.

Fig. 6 plots $\bar{N}$ as a function of $E_{b} / N_{0}$ for $R_{c}=5 / 6$. The difference between the MC and the adaptive MD is less than one iteration, i.e, less than 85.5 additional additions whereas the original MD needs one more iteration with respect to the MC. The MB needs around two more iterations, or 171 additional additions, with respect to the MC.

On the other hand, Table II provides the ML-detection complexity per coded bit for 4-QAM and 16-QAM modulations. For a code rate $R_{c}$, the complexity per information bit is equal to the one per coded bit divided by $R_{c}$. Table II shows that the MC suffers from a high complexity, the MD has a moderate complexity while the MB and MA have a low complexity. Taking into consideration a number of quantization bits $q$ per floating point number, one multiplication can be done by $q-1$ additions. Therefore, the ML-detection introduces the major part of the receiver complexity especially for high order modulations. And, the gain in terms of number of iterations does not compensate for the complexity of the ML-detection.

\section{CONCLUSions}

In this paper, we have presented a non-asymptotic design criterion for MIMO STBC based on the BMI maximization at a specific target SNR. Under the proposed criterion, we have optimized the rotation angle of the $2 \times 2$ low MLdetection complexity MD STBC [5] for a wide range of
SNRs leading to the proposal of an adaptive matrix D based STBC. Practical communication systems promote the usage of low complexity receivers. Thus, the adaptive MD STBC could be a good choice trading off performance and MLdetection complexity. Moreover, the introduction of adaptive STBCs is straightforward especially for systems with adaptive modulation and coding as the appropriate angles are chosen with each coding rate, modulation order and $E_{b} / N_{0}$.

\section{ACKNOWLEDGMENT}

This work was funded by the council of the Region Bretagne.

\section{REFERENCES}

[1] S. M. Alamouti, "A simple transmit diversity technique for wireless communications," IEEE J. Sel. Areas in Commun., vol. 16, no. 8, pp. 1451-1458, Oct. 1998.

[2] P. W. Wolniansky, G. J. Foschini, G. D. Golden, and R. A. Valenzuela, "V-blast: an architecture for realizing very high data rates over the rich-scattering wireless channel," in Proc. IEEE Int. Symp. on Signals, Syst. and Electronics (ISSSE), Sep. 1998.

[3] L. Zheng and D. N. C. Tse, "Diversity and multiplexing: a fundamental tradeoff in multiple-antenna channels," IEEE Trans. Inf. Theory, vol. 49, no. 5, pp. 1073-1096, May 2003.

[4] V. Tarokh, N. Seshadri, and A. R. Calderbank, "Space-time codes for high data rate wireless communications: Performance criterion and code construction," IEEE Trans. Inf. Theory, vol. 44, no. 2, pp. 744-765, Mar. 1998.

[5] S. Sezginer and H. Sari, "Full-rate full-diversity $2 \times 2$ space-time codes of reduced decoder complexity," IEEE Commun. Lett., vol. 11, no. 12, pp. 973-975, Dec. 2007.

[6] J.-C. Belfiore, G. Rekaya, and E. Viterbo, "The golden code: a $2 \times 2$ full-rate space-time code with nonvanishing determinants," IEEE Trans. Inf. Theory, vol. 51, no. 4, pp. 1432-1436, Apr. 2005.

[7] K. P. Srinath and B. S. Rajan, "A low-complexity, full-rate, full-diversity $2 \times 2$ STBC with golden code's coding gain," in Proc. IEEE Global Telecommun. Conf. (GLOBECOM), Dec. 2008.

[8] G. Caire, G. Taricco, and E. Biglieri, "Bit-interleaved coded modulation,” IEEE Trans. Inf. Theory, vol. 44, no. 3, pp. 927 -946, May 1998.

[9] J. Hagenauer, "The EXIT chart-introduction to EXtrinsic Information Transfer in iterative processing," in Proc. European Signal Processing Conf., Sep. 2004.

[10] "IEEE 802.16-2005: IEEE standard for local and metropolitan area networks - Part 16: Air Interface for Fixed and Mobile Broadband Wireless Access Systems - Amendment 2: Physical Layer and Medium Access Control Layers for Combined Fixed and Mobile Operation in Licensed Bands," Feb. 2006.

[11] A. El Falou, C. Abdel Nour, C. Langlais, and C. Douillard, "Low decoding complexity STBC design for turbo coded broadcast transmission," in Proc. IEEE Int. Symp. on Broadband Multimedia Syst. and Broadcast. (BMSB), Jun. 2011.

[12] M. Tao and R. S. Cheng, "Improved design criteria and new trellis codes for space-time coded modulation in slow flat fading channels," IEEE Commun. Lett., vol. 5, no. 7, pp. 313-315, Jul. 2001.

[13] D. Kbaier, C. Douillard, and S. Kerouedan, "Reducing the convergence loss of 3-dimensional turbo codes," in Proc. IEEE Symp. on Turbo Codes \& Iterative Information Processing, Sep. 2010. 